\title{
Social Media as Social Transition Machinery
}

\author{
OLIVER L. HAIMSON, University of Michigan School of Information, USA
}

\begin{abstract}
Social media, and people's online self-presentations and social networks, add complexity to people's experiences managing changing identities during life transitions. I use gender transition as a case study to understand how people experience liminality on social media. I qualitatively analyzed data from transition blogs on Tumblr $(n=240)$, a social media blogging site on which people document their gender transitions, and in-depth interviews with transgender bloggers $(n=20)$. I apply ethnographer van Gennep's liminality framework to a social media context and contribute a new understanding of liminality by arguing that reconstructing one's online identity during life transitions is a rite of passage. During life transitions, people present multiple identities simultaneously on different social media sites that together comprise what I call social transition machinery. Social transition machinery describes the ways that, for people facing life transitions, multiple social media sites and networks often remain separate, yet work together to facilitate life transitions.
\end{abstract}

CCS Concepts: • Human-centered computing $\mathrm{HCI}$ theory, concepts and models • Human-centered computing $\sim$ Collaborative and social computing theory, concepts and paradigms $\cdot$ Human-centered computing Social media • Human-centered computing Empirical studies in collaborative and social computing

\section{KEYWORDS}

Social media; social network sites; life transitions; identity transitions; online identity; Tumblr; Facebook; transgender; non-binary; LGBTQ.

\section{ACM Reference format:}

Oliver L. Haimson. 2018. Social Media as Social Transition Machinery. Proc. ACM Hum.-Comput. Interact., Vol. 2, No. CSCW, Article 63 (November 2018), 26 pages. https://doi.org/10.1145/3274332

\section{INTRODUCTION}

Major life transitions can be difficult to present and disclose on social media. The nature of many social media sites, on which people amass large collections of friends and content over long periods of time, privileges life trajectories in which one's past, present, and future identities remain the same over time. However, many people's identities are not static. A large body of social computing literature has shown the ways that social media sites introduce challenges in interacting online and presenting changing identities during life transitions such as relationship breakups and divorce [38,59,71], job loss [18], transition into and out of military cultures [29,75,76], pregnancy loss [4], and death in one's network [15].

I use gender transition as a case study to understand how people experience life transitions on social media. Gender transition allows insight into these phenomena in a way that other major life changes do not, yet also enables an understanding of social media that can help people facing

Author's address: Oliver L. Haimson (haimson@umich.edu), Univ. of Michigan School of Information, Ann Arbor, Michigan, USA.

Permission to make digital or hard copies of all or part of this work for personal or classroom use is granted without fee provided that copies are not made or distributed for profit or commercial advantage and that copies bear this notice and the full citation on the first page. Copyrights for components of this work owned by others than the author(s) must be honored. Abstracting with credit is permitted. To copy otherwise, or republish, to post on servers or to redistribute to lists, requires prior specific permission and/or a fee. Request permissions from Permissions@acm.org.

2573-0142/2018/November - ART63 \$15.00

Copyright is held by the owner/author(s). Publication rights licensed to ACM. https://doi.org/10.1145/3274332

Proceedings of the ACM on Human-Computer Interaction, Vol. 2, No. CSCW, Article 63, Publication date: November 2018. 


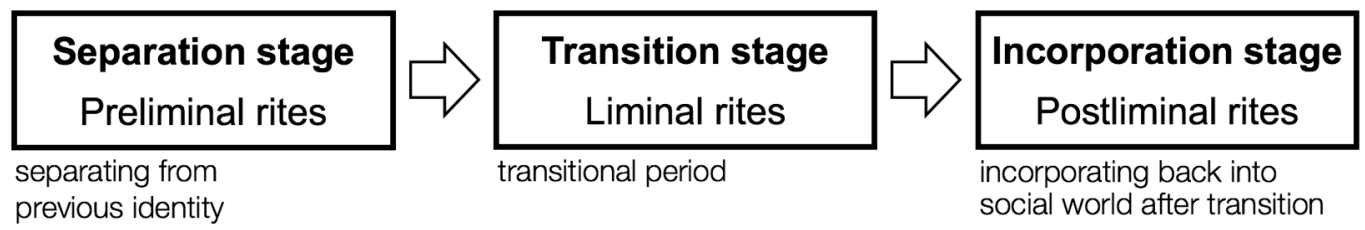

Figure 1. Van Gennep's [33] liminality framework.

life transitions more broadly. Few other life transitions encompass such potentially drastic, numerous, and complex identity changes over time (e.g., name, gender, appearance). Yet importantly, not only should we study transgender experiences as a case study of life transitions more broadly; it is also critical to specifically understand transgender ${ }^{1}$ people's complex experiences. I examined trans people's transition experiences using content analysis of data from transition blogs on Tumblr ( $n=240$ blogs), a social media blogging site on which people document their gender transitions, and qualitative analysis of in-depth interviews with a subset of bloggers $(n=20)$.

In this work, I draw from van Gennep's [33] liminality framework (see Figure 1), along with Turner's [91,92] and Bridges' [14] works which have used this framework in different contexts throughout the $20^{\text {th }}$ century. The word liminality means a sort of threshold that a person goes through during a life transition, and it is used to describe life changes as a series of stages that involve rites of passage. In van Gennep's [33] framework, life transitions begin with the separation stage, in which a person separates from their previous identity - and in the cultures that he studied, people were actually physically removed from their networks during this stage. This is followed by the transitional period, a stage which both Van Gennep and Turner characterized as neutral, in which they described people as identity-less or "a blank slate" $[33,91,92]$. In the final stage, the incorporation stage, the person is incorporated back into a social world after the transition. These stages are not as well-defined and easily delimited as it appears in this framework [52], but this is a way to understand the patterns that occur during life transitions. Van Gennep's [33] and Turner's [91] works were anthropological studies in small-scale, non-Western, non-technologically advanced societies. Yet, they identified patterns that hold up across all societies [87]. But it makes sense that liminality patterns would be different in some ways in networked societies in the present time, particularly given technological advancements like social media. My work builds on this liminality framework, and asks: how does liminality work in social media contexts? While my larger study [37] encompasses the full liminality framework and maps each liminality stage onto different parts of the transition process on social media, in this paper I focus primarily on the transition stage and what Van Gennep [33] called liminal rites of passage (see Figure 1).

I contribute new knowledge towards understanding what liminality means on social media by highlighting the ways social media enable people to embody multiple identities and exist in multiple networks simultaneously during life transitions, rather than remaining neutral (as posited by previous literature about liminality [33,91,92]). I articulate the important distinction between liminality and neutrality, and argue that characterizing those undergoing life

\footnotetext{
${ }^{1}$ I use Stryker's [86] definition of transgender as a term that refers to "people who move away from the gender they were assigned at birth, people who cross over (trans-) the boundaries constructed by their culture to define and contain that gender." I use "trans" for the remainder of this paper to refer to the broad transgender population.
} 
transitions as "neutral" or "identity-less" is dangerous because it dehumanizes people during what is already a vulnerable time. I detail my argument that reconstructing one's online identity is a rite of passage, and conceptualize these findings by introducing the concept of social transition machinery. Social transition machinery describes the ways that, for people facing life transitions, multiple social media sites remain separate and serve different purposes, yet work together to facilitate life transitions. For example, in this study, Tumblr is social transition machinery for intense identity concentration and transition work, while Facebook is social transition machinery for converting a transition into an event. This social transition machinery lens enables new understanding of how people experience liminality on social media.

\section{BACKGROUND AND RELATED WORK}

I review related work on identity and life transitions, liminality and social media, and trans identities and experiences online, then provide background on Tumblr transition blogs.

\subsection{Identity and Life Transitions}

Transition can be defined as "a process of convoluted passage during which people redefine their sense of self and redevelop self-agency in response to disruptive life events" [52]. A life transition is a life change that impacts a person's life deeply over time and involves "reconstruction of a valued identity;" all transition includes change, but not all change is transition [52]. Schlossberg [73] argued that "a transition is a transition only if it is so defined by the person experiencing it." In sum, life transitions are personal, contextual, and alter people's identities [34]. People experience "universal and inevitable" predictable life transitions as they grow older (e.g., from youth to adulthood, from the work force to retirement) [79], but many also experience life transitions that are less common and more unpredictable, whether for themselves or for the people around them (e.g., gender transition, chronic illness diagnosis) $[34,40,52]$. While some studies characterize transition as linear and with a distinct beginning and end, others argue that transition is not linear nor a series of steps, but instead can move forward, backward, and cyclically [34,47,51,52]. Common across transition experiences are dislocation, disorientation, disruption, and a need to find a new way of life that incorporates the changes one has experienced [52]. People tend to deal with life transitions better when they have social support, actively engage with the transition, are well-informed, and after substantial time has passed, among other factors [34].

Sociologists have characterized identity transitions in several unique ways, each of which are useful in considering changing identities on social media. Burke [19] conceptualized life transitions as interruptions to identity processes, brought about by a disruptive life event. He argued that identities resist change and are relatively stable, but do change over time in response to disruptions or identity discrepancies [19,20]. Ebaugh [31] theorized life transitions as role exit: "the process of disengagement from a role that is central to one's self-identity and the reestablishment of an identity in a new role that takes into account one's ex-role." Life transitions often involve unlearning expectations associated with a previous role, while simultaneously learning expectations of a new role [31]. Socialization into a new role is accompanied by an identity shift into that new role, yet often involves tension between one's past, present, and future identities because people are uncomfortable fulfilling expectations for multiple roles at once [31].

Previous research has found that health professionals, such as counselors, nurses, and doctors, can better help their patients if they understand transition processes better [16,52], and 
I argue that the same is true of technology designers. To design technology that supports the myriad life transitions that people face, technology designers must understand transition. In this paper, I provide some of that vital knowledge.

\subsection{Liminality and Social Media}

A small set of previous literature has examined liminality and rites of passage on social media. I briefly discuss each of these works to provide context for my own argument for how liminality works on social media. I extend previous work by providing a new theoretical argument, via the concept of social transition machinery, that liminal identity is multiple rather than neutral, and that reconstructing online identity is a rite of passage.

Most relevant to the current study, several studies have focused on life transitions, social media, and liminality. First, Semaan et al. [76] found that in the context of veterans returning to civilian life, information communication technologies (ICTs), including social media, enable people to re-integrate into society by developing identity awareness and connecting to similar others to understand post-military life and receive support. Veterans often relied on online networks of other veterans in separate digital spaces from their primary online social networks [76]. This separation can be especially helpful because veterans often face difficulties when cultural norms or social worlds overlap between previous and current identities [13].

Next, De Choudhury and Massimi [25] found that people use social media during marriage engagement to project this liminal stage to their networks and make the transition visible. They found that engagement prompted significant linguistic changes on social media [25]. People increased posting in a "rush of excitement" to reach their audience and present their new social role. In this way, people used social media as part of the liminal ritual of exiting one's single identity and entering a new social role as a coupled person [25]. This serves as evidence that social media is an important means of rites of passage, as I argue in this work.

On a more societal level, crisis informatics research has established that after major life disruptions (e.g., war), people use ICTs to recover from the disruption, restore a sense of normalcy, maintain social norms and develop new ones, rebuild societal structures, and create safe online spaces to express identity and communicate with others both locally and globally $[3,77,78]$.

Most previous social media work focused on liminality has conceptualized liminality differently than I do here, largely due to different research questions and approaches. For instance, Papacharissi [62] theorized online citizen journalism as a liminal space.

Several researchers have considered spaces between physical and virtual worlds to be liminal. Ringland [69] discussed the ways in which moments when users sign up for virtual worlds and social media sites are liminal spaces between physical and virtual worlds. Similarly, Herwig [46] considered sign-up procedures on social media as a liminal stage where a person transitions from one social status to another via an interface. Herwig [46] also posited that people go back and forth from mainstream society to a liminal community when they log in and out of Twitter. Yet in contrast with the time when that paper was written (2009), in the present, people rarely "log out" of social media sites, and thus exist simultaneously in mainstream society and in liminal communities.

In online forums (pre-social media sites like Facebook and Tumblr), Madge and O'Connor [55] theorized liminal space as a simultaneous existence in online and physical space while between life stages. They found that new mothers used the Internet during this liminal phase in their lives to explore their new identities as mothers and to craft new selves [55]. Yet in 2005, 
when that research was conducted, Madge and O'Connor [55] found that women substantially decreased their Internet usage after this transitional stage was over. This is no longer true; as evidenced by transition bloggers' experiences and by pervasive social media use over time in the population more broadly [36], people stay online throughout and beyond life transitions. On social media, some people experience transitions in their own lives as tied to movements from one social media platform to another [70]. For example, Robards [70] found that in 2007-2010, many characterized their move from MySpace to Facebook as a transition from youth to adulthood. In this case, what was actually a broader cultural shift from MySpace to Facebook appeared to participants as a personal means of facilitating a life transition by changing social media platforms and networks [70]. Such transitions left digital traces, and were highly visible rites of passage [70]. Similarly, during gender transition, people make highly visible changes to their online identities, in the form of trans identity disclosures and profile changes on Facebook. Yet unlike the transition shift from MySpace to Facebook, in the case of Tumblr and Facebook people do not leave one site and go to another. As I will show in this work, transition bloggers exist simultaneously and present multiple versions of self on Tumblr and Facebook.

\subsection{Trans Identities and Experiences Online}

Relationships between the body, the self, technology, and gender are far from simple. Gender, even though it is a social construction [21], is still based on relation to a physical body [84]. Using an avatar different from one's physical world gender was in the 1990's, and remains today, common in online spaces like virtual worlds [84]. Online spaces can be important means for people to explore identities that they may not yet be comfortable presenting in the physical world - this is what Bruckman [17] called "identity workshops." Stone [84] argued that online, where identity performance, play, and disruption are possible and even encouraged, the "transgendered [sic] body is the natural body." Trans identities embrace both the multiple, as in embodying multiple genders (whether in the same or in different temporalities), and the liminal, as in embracing transition and crossing the boundaries between genders [84].

However, in recent years, many online spaces have begun to substantially "other" trans identities. This has been accomplished primarily through binary gender classification schemes (Male/Female) [9] and enforcement of "one person per account" policies (e.g., on Facebook). On social media sites like Facebook that forbid pseudonymity and multiple accounts [42], gender transition is often difficult [40]. Presenting oneself online using a post-transition gender, name, and appearance often requires mass trans identity disclosure [40]. Such platforms are challenging to those who need to engage in experimentation and exploration to find their new gender identity [39]. Even after transitioning, digital footprints representing a past gender, name, and appearance may remain on one's profile [41]. While much has been done to address some of these challenges around self-presentation, such as Facebook's custom gender options, there remain some problematic programmatic decisions. Bivens [8] described how Facebook's increased gender options only exist at surface level and are still coded as binary in the database.

Despite barriers to experimenting with identity in some online spaces, other online spaces can be important arenas for identity exploration. Because attributes like appearance, name and gender presentation are sometimes flexible, digital spaces can be important places for identity exploration without some of the barriers present in the physical world [39,90]. YouTube is a common site for trans identity exploration, and transition vlogs function as a combination of autobiography, diary, and means of social connection $[66,67]$. 
Many trans people turn to Tumblr, a site that allows pseudonyms and multiple accounts, and where one's network is not necessarily linked with one's pre-transition network, to experiment with and express trans identities. Tumblr's affordances enable "low-risk self-presentation where individuals can try identities out... to a wide, unpredictable swath of other users whose identities are also often temporary and not clearly tied to a real-world persona" [26], which is especially useful for those in transition. Gonzalez and Fritz [35] argued that to best serve trans populations, site design should prioritize flexibility (e.g., in control of information) and foreground intangibles (e.g., political values, support). In this sense, Tumblr's reputation as a queer and gender fluid-friendly space [93] is notable. For many users, Tumblr is a queer space both in terms of queer theory (which challenges dominant gender categories and posits identity as socially constructed, fluid, and ambiguous rather than fixed or essential [21]) and in terms of being populated by queer/LGBT communities [22]. Fink and Miller [32] argued that Tumblr is a space where those belonging to trans and queer subcultures can represent their identities and cultures on their own terms, rather than relying on the mainstream media to represent them. Tumblr is also an important place for trans people to find and share information [2]. Trans people have unique health needs, and thus different information seeking practices, than the general population, and even other LGBQ people; thus health information seeking and sharing is a major focus of the trans community on Tumblr [45].

Many features and affordances contribute to Tumblr's prevalence of trans users and its reputation as a safe place for trans online communities. Users' ability to be pseudonymous, maintain multiple identities and blogs, and the site's unstructured, flexible profiles (on which people can choose which parts of themselves to display, and how) allow people to express nonnormative identities [61,68]. Harassment is de-incentivized on Tumblr because comments (called "replies" on Tumblr) are less visible than on other social media sites - somewhere in between public and private [53] - making the site a relatively safe space for marginalized populations [68]. The site's Ask box is a way to build community around marginalized genders and lets people know they are not alone [61]. Tumblr's visual design enables identity complexity [93], and with its tagging system people can denote gender and find others with similar genders [24]. Finally, the site facilitates nonlinear, impermanent, and multiple trajectories of content reblogged across the site and personal narratives [22,32,93]. These features lead to an openness in discussing stigmatized issues [64,74], which likely influences the prevalence of transition blogs on Tumblr.

Ahmed [2] argues that HCI literature often either ignores trans people, or treats them theoretically rather than as people to be designed for. In response, she introduced the concept of trans competent interaction design, as a "starting point toward anti-oppressive design practices regarding gender" [2]. Among other goals, trans competent interaction design recognizes that design decisions can disproportionately harm trans people; makes changes to minimize this harm; and understands that users have unique identities that may change over time [2]. Recent HCI work has moved towards trans competence by understanding trans people's perceptions toward automatic gender recognition systems, which is disproportionately harmful for trans users [43], considering how to account for non-binary gender variation in online communities [50], and designing an application to support safe restroom access for trans people [7]. Building on previous research about gender transition on social media [39-41], with the current research I address the trans competent interaction design [2] agenda by providing in-depth analysis about the contexts in which trans people use social media during gender transition.

\subsection{Tumblr Transition Blogs}

Proceedings of the ACM on Human-Computer Interaction, Vol. 2, No. CSCW, Article 63, Publication date: November 2018. 
Transition blogs are a genre of Tumblr blog in which people document their gender transition. Commonly, these blogs include diary-like entries discussing social, medical, and legal aspects of transition: discussion of the coming out process and resulting acceptance, support, and/or rejection, physical and mental changes, medical procedures such as hormone replacement therapy and gender reassignment surgeries, and name and document changes. Transition blogs include text, photos and/or videos documenting changes over time. Transition blogs also often include personal day-to-day anecdotes not related to gender transition or trans identity. Though blogs resemble diaries in some ways, blogs are different because they are inherently social [60]; transition bloggers exist within a network of other bloggers, many of whom follow and interact with each other. Transition blogs are different from most other types of Tumblr blogs, which are often more about curation and reblogging others' content than documenting one's own personal experiences [74,96].

Tumblr is not the only platform trans people use to document their transitions; many use YouTube [23,67], and some participants in my study mentioned that people also increasingly use Instagram for this purpose. Tumblr is unique because many transition blogs contain substantial text content, unlike transitions as documented on YouTube and Instagram which include primarily visual content.

\section{METHODS}

I next detail my methods, which involved collecting and analyzing data from Tumblr transition blogs and conducting and analyzing interviews with a subset of bloggers. This study was approved by University of California, Irvine's Institutional Review Board.

\subsection{Tumblr Transition Blogs Data Collection}

Using Tumblr's API [89] and the PyTumblr API client [65], I collected text data from 240 transition blogs starting with each blog's first post (some beginning as early as 2009 and some as late as 2016) and ending on January 4, 2017. My data collection approach appears to be in line with Tumblr's API License Agreement, which gives permission to "manipulate information and Content from the Tumblr Firehose for the purposes of non-public analysis and search" [88]. I selected the blog sample by first searching on Tumblr using the tags "transition blog," "transition," "mtf transition," and "ftm transition," and then searching using relevant tags that emerged within this initial sample, such as "girlslikeus," "hrt blog" (hormone replacement therapy), "hrt transition," "my transition," "nonbinary transition," "personal," "tpoc" (trans person of color), and "transition timeline." I selected blogs that met the following criteria:

1. Included text content more than 10 words long (to enable meaningful content analysis)

2. Focused on the person's gender transition or on personal content, rather than being a blog by a trans person about another topic (e.g., fashion, politics)

3. Bloggers appeared to be, or stated that they were, 18 or older

Using my Tumblr account, I then "followed" each blog currently in the sample. I examined all blogs listed in my account's list of "recommended blogs" to follow. The recommendation algorithm gave mostly accurate and relevant recommendations, and I added all recommended blogs that met my criteria to the sample. I continued this process for approximately one month before settling on a final sample of blogs. Different blogs were active and inactive during different time periods throughout 2009-2016. 
Because of the sensitive, often de-identified, and public nature of the content, I used an optout approach to data collection. ${ }^{2}$ I messaged each blogger using Tumblr's messaging system, briefly described the study, and asked them to respond and specify if they wished to opt out of data collection. I attempted to message a total of 257 bloggers. 121 (47\%) bloggers responded: $106(41 \%)$ of the total initial sample explicitly gave permission to collect their data, $16(6 \%)$ opted out, and three (1\%) asked for more information but did not opt in or out. I answered all questions that bloggers asked about the study and data collection. I collected data from the 241 people who did not explicitly opt out of data collection. I removed one blog that did not include sufficient text content. This resulted in 240 blogs and 84,481 total posts.

Tumblr users can post content in nine ways: text, photo, quote, link, chat, audio, video, answer of a question asked of that blogger by another user ("asks"), and as a "reblog" of another user's post. I collected text content associated with these post types if it was more than ten words long: text content from text, chat, and quote posts, answers from asks, photo and video captions, text accompanying links and audio clips, and text annotating a reblog. I did not collect photos, images, or visual content of any kind.

\subsection{Tumblr Transition Blogs Data Description}

The full dataset included 84,481 posts, with average word count of 71.38 (median $=33, S D=$ 124.70). On average, each blogger posted 367 total text posts that met my data collection criteria (median $=76, S D=814.46$ ), had been blogging for almost two years (mean $=646$ days, median $=$ 530 days, $S D=515.19$ days), and posted roughly three times per week (mean $=0.43$ posts per day, $S D=0.67$ posts per day). Although I did not collect demographic data from bloggers, I found much of this information in their blog descriptions. Most bloggers (95\%) prominently stated or implied their gender and many $(42 \%)$ their age. When placing each blogger into the most prominent gender category that they displayed on their blog (with the caveat that some identified as more than one gender, e.g. trans woman and non-binary), bloggers in the dataset were $47 \%$ female-to-male/FTM/trans men, $46 \%$ male-to-female/MTF/trans women, and $7 \%$ nonbinary (people whose genders are multiple, fluid, or something other than male or female). Like the Tumblr platform more broadly [83], the sample skewed young, with $63 \%$ of bloggers in the $18-24$ age range, $30 \% 25-34,7 \% 35-44$, and $<1 \% 45$ or over. Because most bloggers (93\%) did not specify race or ethnicity information on their blogs, I do not report those demographics here.

\subsection{Tumblr Transition Blogs Data Analysis}

My first step was exploratory, in which I immersed myself in the data. I began by closely reading a diverse sample (i.e., bloggers with a range of different genders, races/ethnicities and ages) of 10 transition blogs in their entirety. I had been following trans content on Tumblr for several years prior to this study, but became more immersed in Tumblr transition blogs when this study began. For two months in Fall 2016 I spent approximately one hour each day reading content posted on transition blogs and examining how transition bloggers and their audiences interacted with each other on Tumblr. This enabled me to understand what types of content people posted, how Tumblr's features and affordances work, and how networks of trans

\footnotetext{
${ }^{2}$ An opt-out approach was ethically appropriate given that I paraphrased blog post quotes to reduce traceability, the data was public, some bloggers may not have logged in to view messages or may have chosen not to respond to my message, and the benefit of analyzing a large rich dataset outweighed the minimal potential harm to participants.
} 
bloggers interact. During this phase, I took frequent notes and wrote memos daily to capture my observations.

I then conducted qualitative content analysis [48] on 3,200 Tumblr posts that I manually coded while building a machine learning classifier, which was part of a different segment of this project (not included in this paper). As I read through each post, I took note of content that was relevant to liminality and social media, as well as topics related to the larger study (namely trans identity disclosure, emotional wellbeing, and social support), and other phenomena of interest that emerged from the data. I did not assign codes to every post, as many of them were not related to the study's research questions. I used a combination of conventional content analysis (allowing codes to emerge from the data) and directed content analysis (coding based on an existing theory or coding scheme) [48]. For the directed coding, I used the categories that emerged from my in-depth inductive analysis of the interview data (described in Section 3.5). The coding scheme that I developed from the interview analysis mapped well to the Tumblr posts. I analyzed Tumblr data concurrently with interview data in an iterative process to provide more depth into my understanding of the phenomena of interest.

\subsection{Interviews Data Collection and Participant Description}

I interviewed 20 bloggers whose data were part of the larger Tumblr transition blog dataset. When selecting participants to interview, I took into account variations in gender, race/ethnicity, and age, to ensure that I had a diverse sample demographically. After determining a set of potential interview participants, I contacted each of them using Tumblr's messaging system to request interviews, and scheduled interviews with those who agreed to be interviewed. Interviews were conducted via each participant's preferred method of video chat ( $n$ $=19)$ or phone call $(n=1)$, and lasted on average 60 minutes $(S D=13.8$ minutes, range: $40-88$ minutes). Interviews were audio-recorded and transcribed for data analysis. Each participant received a $\$ 25$ Visa gift card as compensation for their time.

The sample of interview participants was 50\% trans women, $35 \%$ trans men, and $15 \%$ nonbinary trans people. $65 \%$ of participants were white, $15 \%$ black, $15 \%$ Asian, and $10 \%$ Hispanic/Latinx (percentages add up to greater than $100 \%$ because some participants were of multiple races/ethnicities). The average age was 26.65 ( $S D=7.02$ years, range: $19-43$ years old). 18 participants were American (though one was currently residing in Europe) and two were Canadian.

Interviews were semi-structured, to enable me to further delve into topics that were most salient to participants. I asked participants about trans identity disclosure broadly; social media use broadly; trans identity disclosure on social media; emotional wellbeing throughout transition; social support; perceived relationships between disclosure, emotional wellbeing, and social support; perceptions of and motivations for using Tumblr; and perceptions of and motivations for using Facebook, for those who were Facebook users (all but one). I revised the interview questions protocol four times throughout the interview process as I learned more through data analysis. I analyzed interview data concurrently with data collection, and reached data saturation (i.e., no new codes or themes emerged) after approximately 15 interviews; yet I continued to conduct interviews in case additional themes emerged.

\subsection{Interviews Data Analysis}

I approached the dataset of transcribed interviews with an initial interest in liminality and social media, as well as disclosure, emotional wellbeing, and social support throughout the gender 
transition process as documented on Tumblr (the topics of the larger study of which this paper is part), but analyzed the data using an inductive open-coding approach [85]. I coded all interview data using line-by-line analysis, allowing codes and themes to emerge from the data [85]. I conducted axial coding iteratively throughout the coding process to organize and refine codes and themes and understand how they connected to each other [85]. I used frequent memoing as an analysis technique to make sense of the emerging codes and connections between codes and themes. Throughout the analysis process, I discussed emerging themes, and connections between codes and themes, on a regular basis with two other researchers.

\subsection{Limitations}

A primary limitation of this work is that due to the recruitment criteria, all participants were avid Tumblr users. Thus, this paper's results may not generalize to those who do not use Tumblr, those who are more invested in other sites (e.g., Facebook, Twitter), those who document life transitions elsewhere, or those who do not document their transitions. Many of the people I interviewed talked about themselves as "open," an "open book," etc. This may be a result of the sample - it may be that people who write transition blogs are more open and willing to share personal information than other people. Comparing transitional populations who do and do not blog about life transitions is an interesting area for future work.

Another bias to the interview sample is that those people who are most distressed may have been less likely to respond to my interview requests. Those who are depressed or even suicidal (which is prevalent among trans populations [49]) may not have the emotional bandwidth to respond to a message from a researcher on Tumblr, or to spend emotional energy on an interview. Those who were doing worst emotionally may not be in my interview sample, but my analysis of blog data did still capture their experiences.

\section{RESULTS}

During gender transition, Tumblr transition bloggers presented multiple identities simultaneously on different social media sites as a mechanism to keep identities and networks separate. Participants described using Tumblr for transition work, including expressive writing, self-documentation, and identity exploration, in a self-focused yet social online environment. They described using Facebook, when ready, to disclose their trans identity to a large, broad network of "real life" connections.

\subsection{Presenting Multiple Identities Simultaneously}

During transition, people presented multiple identities simultaneously on social media. Almost all interview participants $(n=19$ out of 20) reported using Facebook in addition to Tumblr. Before they had disclosed their trans identity to their Facebook networks, participants described presenting their pre-transition gender and identity on Facebook, while presenting their new or transitional gender on Tumblr. $\mathrm{P} 19^{3}$, who had not yet started living full time as trans, described segmenting her social media personas between networks to whom she was and was not out to as trans:

\footnotetext{
${ }^{3}$ Interview participants are designated with $\mathrm{P}$ and the participant number (1-20). Bloggers are designated with B and the blogger number (1-240).
} 
I feel like [Tumblr] is more representative of who I want to be, so I'd say it's reasonably representative of myself in some ways. It's interesting, because Facebook - at this point, I would definitely say it isn't, and it's a lot more contrived, versus Tumblr, where... everyone that follows me there... I know is supportive, so I don't really feel a desire to craft a persona. I just do whatever I feel like.

While her Facebook profile was somewhat representative of her physical world persona, at least in terms of appearance, P19's Tumblr represented her future, post-transition self. People's particular presentation on the two sites varied, but each person described presenting a pretransition name, gender, and appearance on Facebook while presenting a different name, gender, and appearance on their transition blog.

Multiple identity presentations on different social media sites also involved differences in types of content shared on each site. Participants in my study shared vastly different types of content on Tumblr as compared to Facebook. On Tumblr people shared personal, emotional content, which included both positive and negative emotions. Due to my inclusion criteria, all participants shared trans-related content on Tumblr, yet while some shared trans-related news articles on Facebook, most did not share personal transition updates on Facebook. On Facebook, people shared opinions, everyday content, life updates, and professional or work-related content. Several content types spanned both sites, including everyday content, political content, art, funny things, and content related to people's interests.

Social media allow multiple virtual embodiments, and thus create possibilities for people to exist in multiple spaces and multiple social environments at once. One's Facebook profile continues to represent a version of self to one's Facebook network, even while one presents identity differently and engages in transition work on Tumblr.

\subsection{Keeping Identities and Networks Separate}

Participants tended to keep their identities and networks separate between Tumblr and Facebook during gender transition. When asked about overlap between these networks, P1 said, "they are completely separate for me. My Facebook and Tumblr are 100\% different." For some bloggers, this network separation made Tumblr feel anonymous, which in turn allowed more openness. B75 described on her blog, "Tumblr being fairly anonymous (mostly because it isn't linked to Facebook) is a really good tool for people everywhere to express themselves. " The openness enabled by separation from one's Facebook network created the conditions necessary for many bloggers to disclose personal information and receive social support.

Some who did have overlap between their Facebook and Tumblr networks described this overlap as being uncomfortable, even if the people in common between the two sites were also trans. For example, P4 said:

There is a little bit of overlap and initially it was difficult for me because a few of my Facebook friends also happened to have a Tumblr and... I started being suggested [in the suggested friends list] because they are looking at trans things... That was very weird because it had felt, even though I was putting my face and my name out there it still felt pretty anonymous for a while and then a couple friends found it and they would comment on there. It was weird because I talked about things there that I wouldn't on my Facebook like confirmation surgery.

Even though bloggers knew they were not completely anonymous on Tumblr, the separation from their Facebook network made Tumblr seem anonymous. Conflict often occurred when

\footnotetext{
${ }^{4}$ I paraphrased some blog posts to reduce traceability and to maintain bloggers' privacy, as is common practice in social computing research. Those blog post quotes that were not traceable via Google search are left as is. I revised blog post quotes for spelling, grammar, and readability as necessary.
} 
audiences overlapped between the sites. For example, P8's father reacted negatively after reading a Tumblr post where she discussed him in a negative light. When the boundary between different networks was compromised, as in P4's case when her Facebook friends found her on Tumblr, or when P8's father read her Tumblr blog, sharing intimate content became more difficult.

Other bloggers struggled with how much they wanted their identity to overlap between Tumblr and elsewhere online. B87, a programmer, wrote:

I feel a bit conflicted about whether or not I should post here about the things that I'm programming. I like the idea of having separate internet identities instead of tethering everything together. I can't even begin to imagine why some people even go so far as to tie everything to their meat space name. Why would you want to be stuck carrying around your digital baggage and history with you forever? Facebook probably deserves a fair amount of blame for encouraging/training normal people to use real names online. Anyways my point is that I don't want people that know me as a coder to have the chance to judge me by the stuff that I post here and vice versa. But writing software is pretty much the only thing ever I do that could be passed off as being productive. I rarely have much to talk about aside from programming so not writing about it makes this blog a bit pointless.

B87 described some desire to combine separate topics that were both integral to her identity: programming and being trans. Yet she was conflicted about the people involved in each of those identities being combined into one audience. To handle this type of context collapse [57], Tumblr bloggers often segment their content into several different blogs, based on topics - for example, many described having one transition blog and one blog related to an interest or fandom. Yet B87 felt her blog was "pointless" without incorporating her programmer identity into it; thus, segmentation would not be a solution.

When considering network separation in conjunction with the identity multiplicity that social media enables, it becomes clear that during transition people can at the same time stay within and be apart from their pre-transition networks. This is in contrast to the transition stage described in Van Gennep's [33] and Turner's [91] work, in which people were physically separated from their existing networks during life transitions. By allowing networks and identities to be multiple and separate, social media enables people to remain connected to people they otherwise may have had to cut ties with during identity transitions.

\subsection{Tumblr for Transition Work}

Tumblr is an online space that people use during gender transition to engage in transition work. Transition work involves expressive, therapeutic writing, self-documentation, and identity exploration. Tumblr is uniquely suited for these types of activities as a space that is both selffocused yet social.

Using transition blogs therapeutically or as an "outlet" was the most frequent reason interview participants mentioned for keeping blogs, echoing findings from Schiano et al. [72]. P15 said that his transition blog was,

Definitely a huge outlet for me. I'm the type of person that I do hold a lot of emotions in and I don't necessarily share a lot, but when I do share it I know that I feel better about it, so I will spend that personal time to post... my blogs, to kind of just get those emotions out, and to kind of just share what's going on through my head.

As P15 described, and as was true for many others, keeping a transition blog enabled him to feel better over time by processing his emotions through writing. P4 described a similar experience with blogging:

When things are in my head they tend to spiral, and if I can get them out of my head I can deal with them a lot more rationally than emotionally, so [blogging] helped me to do that as well to get that out and look 
at it objectively. Sometimes even I would type out something and even the act of typing it out was therapeutic and I wouldn't feel the need to post it... Sometimes there are posts where I'm feeling this way and it's so negative and it's so bad and then I'll keep typing and I'll go through the process but now that I'm looking at this and I've gotten it out of my brain, I realize that blah blah blah that I got this and I can totally do it. Sometimes it helps me work out those things as well.

Like P4, many participants experienced positive benefits from writing blog content on the Tumblr interface whether they ended up posting that content or not.

Many used Tumblr as a tool for self-documentation, to keep a personal record and track transition progress. P18's transition blog was,

A way for me to track my progress. That was originally what it was meant for, just because I knew that hormones were a slow and painful process when it came down to the change, and it was like, okay, hey, the best way I can do this is just document it as I go.

Similarly, P16 described his blog as a place to "post updates about things going in my transition like with therapy, and getting my letter, and blood work, and all that shit, voice updates. I post all that stuff on there." These and other participants appreciated having a digital archive of their transition progress. Though they did not specifically mention an intended audience for these transition updates, the posts may be intended for a future self to reflect upon. Other bloggers tracked less physical or social transition progress, and instead blogged to document personal growth and change; as B23 put it, "the only theme here is my experience and evolution as a human."

Documenting one's transition in a digital medium like Tumblr was importantly different than tracking the same type of content in a physical format. I asked participants the difference between blogging on Tumblr and keeping a physical diary, expecting that they might mention the social aspects of blogging in an interactive digital space. While some did answer as I expected, several others emphasized the ways a digital medium like Tumblr enabled easy access to content, as opposed to a physical form. P9 said,

Honestly, I just wanted a place where I could put down my feelings and thoughts. It's the most journalistic thing I've ever been able to keep up with... I've tried to do physical journals and diaries and I always lose them. I just wanted a place where I could jot down my own thoughts in the process and whatnot, and I just liked doing the weekly photos just to watch very slow change happen, which is kind of cool.

Digital journaling spaces like blogs are difficult to lose, enable people to access and add to their blog content online rather than digging for a physical object, and facilitate tracking transition progress more consistently. While the digital form may seem more permanent, one could also argue that online writing is less permanent, given that the web space is managed by a company who could be bought out at any time (and was, during the course of this study), making Tumblr content precarious. One participant, P7, expressed concern about Tumblr's potential impermanence. She was in the process of building her own website and transferring her transition blog content there, "just in case Tumblr ever went away, like certain sites like Vine just suddenly disappear." Online journaling allows people to keep track of their changes in a more convenient and accessible format than a physical journal, but which feels precarious for some.

Finally, some participants used Tumblr transition blogs for identity exploration. P6 described how Tumblr affording multiple accounts and pseudonyms makes identity exploration possible without long-lasting consequences: "You can create blogs and then delete them. It does make it easier to explore without it damaging your name or your personal network - just create a new blog." Many trans people use the Internet and social media for identity exploration, as evidenced by a large body of previous research $[39,90,94]$. Yet on Tumblr, they tend to do this prior to starting transition blogs. Identity exploration on Tumblr, and elsewhere online, helps a person find the identity that they later blog about via their transition blog. Transition blogs are often 
used to document how one's identity changes over time, which necessarily entails identity exploration. Yet once a person settles on a particular blog, with a name and url that they stick with over time ${ }^{5}$, that blog tends to be less about identity exploration and more about documenting how that particular identity becomes known and embodied, socially and physically.

Tumblr is unique in its duality as a self-focused space where people spent time alone, and as a supportive community. B34 summarized the therapeutic and self-focused yet social nature of Tumblr in a blog post celebrating reaching 100 followers:

Thank you for following me and my thoughts. This is a place where I can express and share how I feel. It could be a happy or sad post, a joyous experience or an angry rant or I might feel good about myself and want to share that moment. Either way it makes me feel better and thanks for that.

Tumblr is a place where people can share and process their experiences and emotions with an understanding and supportive audience. Yet, the audience is not the most important part. Writing enables self-understanding and emotion-processing in a self-focused yet networked environment.

One's Tumblr audience exists as a complex interplay between self and others, between being alone and being part of a community. "My only friend that knows me and understands me is Tumblr," B104 wrote on her blog, signaling simultaneously a supportive audience, and the lack of an audience. By invoking Tumblr as an individual friend, rather than a group of people, it comes into focus as a space for B104 to write and process emotions on a personal and solitary level. Yet, at the same time, she subtly describes Tumblr as a supportive community. Similarly, P11 described Tumblr as, "My favorite place to be alone. It's like a place to be alone with your thoughts, but in a public way, in a way that feels supported by others." Tumblr is remarkably different from a site like Facebook in this sense; many participants described feeling an obligation on Facebook to be connected to their network. On Tumblr, in part because many bloggers' "real life" connections are not there, people feel little obligation to follow each other or interact. P11 adamantly described not wanting any obligation to interact, and appreciated that on Tumblr people follow her only because they want to, not because of any pressure resulting from "real life" associations. Yet participants who viewed Tumblr as a place to be alone still also considered it a supportive community. The lack of obligation to one's network created a freeing sense of solitude along with the comfort that one's network would support them if that support was needed. In online communities, both the reflexive aspects, such as expressive writing, and the social aspects are important for user engagement [54]. Blogs are novel because bloggers can simultaneously "engage in self-expression in order to build community and... build community in order to cultivate the self" [58].

Van Gennep [33] described how transitional periods sometimes involve autonomy. Tumblr, for many trans people, is a space for autonomy, where people separate themselves in some ways from their existing networks and become part of a new community of other people who are also in transitional autonomous stages. Tumblr's particular combination of features and networks enables the site to serve as a digital space for transitional networked autonomy.

\subsection{Facebook for Transition Disclosure}

While participants in this study used Tumblr for transition work (e.g., expressive writing, selfdocumentation, identity exploration), when they reached a point in their transition trajectories where they were ready to disclose their transition to a large, broad audience of people in their lives, Facebook was the site where many went to do so. Coming out as trans on Facebook is a

\footnotetext{
${ }^{5}$ Bloggers can change their blog name and the associated url as often as they like, and this is a common practice.
} 
pivotal transition moment for many. How people come out on Facebook varies greatly: some make an announcement and keep their current account and friends list, some heavily edit their friends list before making an announcement; some change their Facebook name, gender marker, and/or appearance in profile photos but do not make an official announcement; and some move to a new account entirely [41]. However, regardless of the methods used to partition networks and disclose one's trans identity, participants reported in interviews and on their blogs how coming out on Facebook was a defining moment in their transitions, and often a somewhat

Soooo I CAME OUT!!

to everyone.. full time.. all the time $=\mathrm{D}$

i really cant believe it but its magnificent .. i think im glowing

and i got such a POSITIVE response on facebook.. it was baffling and amazing

\#lgbtq \#mtf \#transgender \#trans girl \#femme \#amab \#gay \#trans \#lesbian \#non binary \#male to female \#transisbeautiful \#girlslikeus \#girls like girls \#trans lesbain \#coming out \#came out \#empty closets \#came out of the closet \#im out \#out. \#full time \#transgender girl 99 notes Nov 18th, 2016

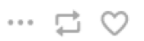

Figure 2. P3's post on Tumblr describing her trans identity disclosure to "everyone" on Facebook (used with permission). final point on their disclosure paths. Many participants characterized Facebook disclosures as meaning coming out to a large audience of primarily "real life" connections, or "everyone" in their lives. Interview participant P3 described her experience coming out on Facebook (emphasis added):

Well, Facebook, yes. That was obviously the big one, that was telling not my close friends that I knew would love me regardless, that was telling everyone... All these people knew at this point and that was huge. I changed my name on Facebook and everything, I just went for it. That was definitely a monumental thing. It really helped by getting that word out to everyone. I had come out in person to quite a few individuals, but to do it to everyone at once was definitely a relief. I had nothing to hide anymore, nothing to be ashamed of... nothing in the closet.

P3's interview quote was similar to her Tumblr post in which she also characterized coming out on Facebook as an important moment of disclosure to "everyone" (see Figure 2).

The Tumblr posts that people posted about their experiences coming out on Facebook give important context about their thoughts, deliberations, and processes around Facebook disclosure, and often also included the text of the Facebook post itself. Many people posted about their Facebook coming out post on Tumblr, both before and after posting it. Before the Facebook disclosure, many posted draft coming out announcements and asked for feedback (e.g., "So this is my coming out post that I'm probably gonna post on my FB page some time tomorrow. Any thoughts?" - B153) or described fears and anxieties around upcoming Facebook disclosures. Afterwards, many posted follow-up posts, often celebratory, describing or including the Facebook disclosure post, responses to it, and the poster's feelings about it. For example, B200 posted: “So on New Year's Eve, I finally came out to the world!! (well Facebook $x D$ ). Here's what I posted: [Facebook disclosure post content]. And so far it's been all positive! I've been worried for nothing $x D$." As these quotes indicate, Tumblr is a place where people reflect on their disclosure decisions and actions, and thus a rich research site for understanding disclosure on social media.

People chose Facebook as a platform for mass disclosure because it was an efficient and official way to disclose trans identity to one's network, similar to how some use Facebook to disclose relationship breakups [38], pregnancy loss [4], and other life transitions. B92 described how making an announcement on Facebook was an efficient means of disclosure and allowed him to circumvent a series of individual conversations. Another blogger (B106) wrote, "Sometimes I just want to come out on Facebook. Post the message, drop the mic, sign out. Come back in a week and see 
what's left. Scorched earth approach." While "scorched earth approach" sounds negative, it is an apt metaphor for coming out to one's entire social network at once. Posting information on Facebook is an efficient means of reaching one's network without having to disclose to each person individually [4], but it may feel untargeted and reckless for some. As B106 described, coming back a week later to see how her network responded, and who remained in her network, was an attractive disclosure approach that may allow her to spend less emotional energy on disclosure.

While in many other life transitions people can choose whether or not to disclose to their Facebook networks, during the transition stage trans people often reach a point where they are no longer able to conceal their physical changes. B3 described on her Tumblr how she was approaching a point in which she may be "forced to come out to everybody:"

So it's getting a bit harder to hide things. Only a bit over 3 months in, but I'm seeing some progress for sure...

I'm just fine if anyone starts questioning my changes, and if I am sort of forced to come out to everybody, I'm okay with that.

Because Facebook allows mass disclosure to one's network, it can be an important tool for people who, like B3, feel that physical changes may soon make disclosure inevitable.

Transition disclosure on Facebook, and online identity changes more broadly, can also help people feel that they are making progress in their transition and moving forward in their new identity. B27 described on Tumblr her experience changing over to new Facebook and LinkedIn profiles:

So, I finally came out to the remaining people back home that I want to keep in touch with. (I was waiting a bit because I was giving family back home in our small town more time to process before it became common knowledge.) Some people friended my new Facebook profile and some didn't. However, no one had anything bad to say and I got a lot of heartwarming responses, so I'm calling it a win. I closed down my old Facebook and LinkedIn accounts, so I think all on-line accounts of the old me are officially dead and gone. It feels really good to be moving forward in life completely as I am!

Moving to new profiles on Facebook and LinkedIn was a pivotal transition moment for B27, after which she described feeling that her online identity was congruent with her new gender identity.

Facebook disclosures usually occur after people tell close family members and the other important people in their lives, with whom they feel a one-to-one conversation is necessary. With these disclosures out of the way, Facebook disclosures are now possible, as P11 described:

As soon as I came out to my dad, I was just - I had told everyone. I made a Facebook post and I started presenting more how I pictured myself in my head, at the time. And I was pretty much just out...

Facebook disclosures are also sometimes prompted by a combination of family disclosures and present or upcoming physical changes. P13 stated that he came out on Facebook " $a$ few days before I was supposed to start T [testosterone hormone replacement therapy], and after I talked to my family, of course."

As this analysis shows, trans identity disclosures on Facebook are pivotal moments for many. While the unique nature of each person's transition timeline makes it impossible to divide gender transitions into before and after time periods, Facebook disclosures often mark a substantial change in which people go from being out as trans to some people in their networks, to being out to "everyone."

\section{SOCIAL MEDIA AS SOCIAL TRANSITION MACHINERY}

Next, I discuss my results in the context of liminality and rites of passage. I argue that reconstructing one's online identity is a rite of passage in our society. I then introduce my social transition machinery lens to provide new understanding of how liminality works and how 
people experience liminality on social media. I close with an argument for the importance of social media site separation.

\subsection{Liminality, Then and Now}

The major work on liminality throughout the $20^{\text {th }}$ Century characterized the transition stage as neutral, or a state of betweenness. Following van Gennep [33], Turner [91,92] described the ways in which liminal people were stripped of identity to become a "blank slate" or a "uniform condition," on which one's new identity could then be inscribed. Turner [91], in a quote that has been cited abundantly in the life transitions literature, wrote that people in the liminal stage are "neither here nor there; they are betwixt and between the positions assigned and arrayed by law, custom, convention, and ceremonial."

In the context of gender transition, while most previous studies have characterized liminality as a neutral state, each gives hints that it is more complex than this. Bolin [10] used Turner's [91] neutrality framing to describe gender transition as "a journey through liminality, where transsexuals are 'betwixt and between,' for they are no longer males and not yet complete women." Yet even so, Bolin [10] described many instances throughout the transition process in which trans women presented as women in certain social contexts, and as men in other social contexts - a practice that caused distress for participants. This signals a liminal state that is more multiple than neither, as I also find in this study. Wilson [95] also described gender transition as a state of betweenness and limbo, yet at the same time discussed the identity multiplicity that occurs during the liminal stage. Participants in that study described feeling neither because gender categorization systems failed to account for their liminal state - not because of an internal sense of lacking identity [95]. More recently, in a survey of trans and non-binary people, Harrison et al. [44] found that $20 \%$ described their current primary gender as "part time as one gender, part time as another," signaling that gender fluidity and oscillation is relatively common. Similarly, Bauer et al. [6] quoted an interview participant as saying, "It's hard to distinguish for me between feeling both male and female and feeling neither." Ambiguity is an important aspect of gender transition, and on a personal level, people cannot always distinguish between a gender identification that is back-and-forth, or one that is between. Turner [92] briefly discussed the notion that those in the liminal stage may experience multiple identities in addition to his primary conception of liminality as a neutral state of betweenness, and acknowledged that identity may be ambiguous and paradoxical during transitional stages. In my argument I do not mean to place any claims on how people self-identify, or imply that people's self-identification is with multiple genders rather than one or none. Instead, I address the ways people present different gender presentations to interact in different social contexts, many of which are facilitated by social media.

While I argue that the transition stage is not neutral, certain aspects of Turner's [91] transition framing are useful to theorize Tumblr transition blog communities. Turner [91] described the ways in which people who are together in the liminal stage form kinship, camaraderie, and social structure as a result of being equal in their neutrality. He used the term communitas to describe these groups of people who experienced transition together, and described them as not only liminal (i.e., in the crevices or "interstices" of societal structure), but also marginal (i.e., on the edges of society) and inferior (i.e., beneath the rest of society) [91].

While the communitas concept [91] cannot as a whole be applied to Tumblr transition bloggers, certain aspects do apply. Namely, Tumblr transition bloggers form a supportive community that embodies kinship and camaraderie and forms a social structure around the 
shared experience of gender transition. Yet transition bloggers are not neutral, liminal, marginal, and inferior in the ways that Turner [91] characterized other communitas. Those embarking on gender transition form bonds based on shared experience and social support, not because of a shared neutrality. While transition bloggers are liminal, this liminality does not place them in the crevices of society (i.e., between two social roles) as Turner [91] described, but rather sees them presenting differently in different social contexts (e.g., Tumblr and Facebook). Trans people are marginalized in the sense that they are othered and treated as outsiders by many, but not in the sense that they exist on the edges of society; rather, they exist in the same social structures as everyone else, though sometimes in multiple forms simultaneously. And finally, though many people may consider trans people to be inferior, those in transition do not generally consider themselves inferior to the rest of society.

A major contribution of the present study is a new understanding of how liminality happens and what liminality means on social media and in relation to online identity. Van Gennep's [33], Turner's [91,92], and Bolin's [10] conceptions of liminality as neutral may have been true for the cultures and time periods they studied, and may also be related to their anthropological disciplinary and research approaches. Yet one primary difference in my study is the element of social media. On social media, whether or not a person is actively using a site or sites, users are always connected to their networks, and always presenting version(s) of self. This presents a double-edged sword: on social media it can be difficult to escape one's social networks, yet at the same time social media enable unique ways to present multiple identities at once. Thus, liminality is inherently different when expressed on social media, because people can simultaneously present two, or more, identities at once, and can segment audiences and networks by using multiple platforms (in this case, Tumblr and Facebook) or account management strategies. Presenting different online selves does not necessarily mean that one identity is more "real" than another [97]; each are genuine in some respects, and performative in some respects. Throughout transition, different online identities' salience shifts, and some profiles may remain only as a stand-in self-presentation for those to whom one has not disclosed their trans identity (e.g., in the case of those who maintain a Facebook profile for their previous gender, for the benefit of extended family members to whom they choose not to disclose). Other profiles are used heavily. In whatever configuration, multiple online versions of self can present different identities simultaneously. Social media enable simultaneous identity multiplicity in ways not possible in the physical world.

\subsection{Rites of Passage and Identity Transformation}

Identity transformation on social media is a rite of passage in our society. Drawing from the liminality literature, I first describe what rites of passage are and what they mean. Rites of passage are "lenses through which to magnify" transition experiences, and to focus on and make visible the liminality patterns (separation, transition, and incorporation) [14]. They are transformative and confirmatory events that enable people to transition from one identity, social status, or social role to another [56,92]. Rites of passage provide structure and meaning and are meant to dampen the stress and anxiety that accompany moving from one social role to another by giving the transition a cultural lens [33]. Rites of passage help people to reconstruct their lives to fall in line with their new identity, and require one to "pass through a socially mediated series of culturally as well as personally meaningful experiences” [56]. Rituals can help people integrate their life transition, and their previous identity, into their life story [82]. Biographical work is a way of coping with life disruptions or transitions by connecting past and 
present life narratives, and can be used along with rituals to move through liminality [82]. Rites of passage are often expressed using symbols and ceremonies. They often happen as part of religions, but religious ceremonies are not the only ways for rites of passage to occur $[10,82,91]$. Rites of passage can involve anything that is meaningful or symbolic to a person [82], and can be technological [10]. Rites of passage may not have concrete, measurable outcomes, given that they are deeply personal and often abstract [82]. They have in common the ability to propel a person forward out of the liminal stage.

Gender transition is a specific type of transition with its own particular phases and rites of passage. While in the past some considered sex reassignment surgery (SRS) the primary trans rite of passage [10], today, as my study demonstrates, trans rites of passage are much more varied and complex. I argue that online identity reconstruction is a modern pivotal trans rite of passage.

Previous literature has often examined rites of passage in small-scale, non-technologicallyadvanced societies that were relatively stable. Van Gennep [33] did not expect rites of passage to generalize beyond these small stable societies, yet taken together the liminality literature published in the century after Rites of Passage was published, it is clear that van Gennep's liminality framework describes a pattern and a sequence that applies to all types of life transitions in all societies [87,91]. While each society and each life transition has complexities and differences in the particular rites of passage performed, the liminal pattern is universal [87].

In modern networked cultures, life transitions are often distressing and disorienting because they do not involve standard rites of passage, and thus can feel meaningless [14,56]. Many traditional societies had standard rituals, or rites of passage, that helped people let go of one life stage and move to another [14]. While modern networked societies have standard rituals for life transitions such as marriage and childbirth, we do not have standard recognized rituals for many other life transitions, such as divorce, career change, transition from college to the workforce, and, of course, gender transition. Drawing from van Gennep [33], Bridges [14] argued that:

without formal transition machinery, such as the old rites of passage, we not only lack the support that traditional people enjoyed but also the powerful concentration that the old rituals provided - a power that took an extended and diffuse time of transformation and converted it into an event.

\subsection{Social Media are Social Transition Machinery for Identity Reconstruction as a Rite of Passage}

I provide a new understanding of social media as what I call social transition machinery. This is informed by Donath's [27] characterization of the computer as a social machine, which is a "communication medium and a setting for interactions, an electronic place to see and be seen" and by Bridges' [14] statement that we lack transition machinery for many life transitions. I argue that social media are these social transition machinery that enable rites of passage. Transition experiences are fundamentally different in networked societies in the present time, particularly due to several factors related to social media. I detail my argument in the context of this study, which involves gender transition on Tumblr and Facebook, but these results are likely relevant to other life transitions and other social media platforms. Social media are social transition machinery and enable rites of passage in two ways: they serve as means of transition work and intense concentration on identity (e.g., on Tumblr), and as a means to convert a transition into an event (e.g., on Facebook). I outline my argument in four steps: 
1. During the transition stage, social media enable people to embody multiple identities simultaneously rather than being neutral.

2. People do not fully remove themselves from their existing networks during life transitions.

a. In this study, they remained connected through Facebook, yet retreated to Tumblr to do transition work.

3. Social media act as social transition machinery during life transitions.

a. In this study, Tumblr is social transition machinery for identity concentration and transition work.

b. In this study, Facebook is social transition machinery for converting a transition into an event.

c. Social support occurs on separate sites as part of transition processes, but in different ways.

4. Combined, these social transition machinery serve as means for those in transition to reconstruct their online identity as a rite of passage.

\subsubsection{Multiple, Not Neutral}

First, social media enable people to present as multiple identities simultaneously, and as such, transition is not a neutral stage. This is in contrast to previous conceptualizations of the transition stage as neutral $[33,91,92]$ or as a time in which one is neither the previous identity nor the new identity. Instead, during transition, people present different versions of self to different networks in different social contexts, on social media sites and in the physical world. It is important to make the distinction between liminality and neutrality because characterizing transitional periods of people's lives as neutral or identity-less is dangerous and dehumanizing. Particularly when many people in transition are already part of marginalized groups or are facing stigma as part of their identity change, adding neutrality as an additional judgement label may be especially harmful. People in transition have just as much identity as anyone else - it is just that that identity is multiple, fragmented, and complex. Changing the cultural narrative of identity transition to account for multiplicity and complexity, rather than characterizing people as neutral, could make great strides in improving wellbeing for people going through difficult changes in their lives.

\subsubsection{Network Fragmentation, Not Removal}

Next, transition no longer involves fully removing oneself from one's existing network, as was common in the cultures described in van Gennep's [33] and Turner's [91] work, but instead involves fragmenting one's online social participation between several different sites. In networked societies in the present time, people remain connected to their networks on one site, and use another site to do transition work. In this study, people used Facebook to remain connected to their existing networks, and used Tumblr for transition work. Yet for other transitional populations, other sites may fill each of these roles.

\subsubsection{Social Transition Machinery and Online Identity Reconstruction as a Rite of Passage}

Because networked societies lack formal rites of passage for many life transitions, for social media users, social media fill this gap by acting as social transition machinery for life transitions. Different social media sites serve as different pieces of social transition machinery that are separate but work together to facilitate life transitions.

For transition bloggers, Tumblr is social transition machinery where people concentrate intensely on their transition and their identity and engage in transition work, such as by 
writing expressively, documenting their transitional journey, and exploring their identity. Bridges [14] characterized the transition stage as a time period in which people should go to an unfamiliar place to think a lot, do little, and act as though they are performing a ritual, in order to focus on the transition process. Bridges' [14] suggestion corresponds with my finding that Tumblr is an important place during transition for people to express emotions (whether happy, lonely, sad) and to be alone. Yet Tumblr enables people to be alone in a networked environment and among a supportive community of similar others.

At the same time, for many in transition, Facebook is transition machinery in which a transition becomes an event. Changing one's online identity on an identified site like Facebook involves a symbolic passage and a change of status from one identity category to another. This can occur in many ways, such as by editing one's Facebook profile with a new name, gender marker, appearance, and sometimes network; by creating a new Facebook profile with these attributes; and/or by disclosing one's trans identity to one's Facebook network. Any combination of these behaviors that signals a gender change to one's network serves as a means for converting a transition into an event, and thus a social and cultural rite of passage. Symbols in rites of passage often reference both one's past and future status [10]. Changing one's gender marker, name, and/or profile photo on Facebook is a symbolic aspect of gender transition rites of passage that involves both one's past and future identity and a shift from the former to the latter. By changing one's online identity, a person signals a life transition to their network and thus converts the transition into an event.

Social support is an important part of social transition machinery that occurs on both of these sites, but in different ways. On Tumblr, transition bloggers form a supportive community of people going through a similar life change, and share advice, information, emotional support, and a sense of belonging. On Facebook, after a person converts their transition into an event, their existing (e.g., pre-transition or "real life") network rallies around that event to provide support in the form of likes, comments, and messages.

Reconstructing one's online identity is a rite of passage. My transition machinery lens provides new insight into how life transitions are conceptualized, and what social media means during life transitions.

\subsubsection{Social Transition Machinery in Other Life Transition Contexts}

Identity multiplicity and network fragmentation have been observed in other life transition contexts, providing evidence that my social transition machinery lens extends beyond the gender transition case study. As just a few examples, Durrant et al. [30] found that tensions between different social roles for new parents meant that people performed different social roles in different online social spaces, such as a Facebook group for new moms vs. one's primary Facebook profile, during this transitional period. Similarly, Semaan et al. [76] found that veterans used veteran-specific ICTs (e.g., RallyPoint, secret Facebook groups) separate from their Facebook networks to connect with other veterans and discuss difficulties transitioning back to civilian life. These are examples of social transition machinery in action.

\subsection{The Importance of Social Media Site Separation}

Understanding social media's role during life transitions provides implications for effectively designing technology that accounts for and supports people during times of transition. Social media technologies can enable people's networks to provide support, and potentially provide 
support themselves, to those who most need it during life transitions. Yet such technologies must be designed to work in the complex and changing contexts people use social media during transitions.

Social media are a series of separate sites that together enable identity reconstruction as a rite of passage; yet at the same time, identity change requires network separation. However, social media sites and services constantly urge users to connect across sites and networks that may be discordant. This brings up difficult technological and social challenges. Transition bloggers' retreat to Tumblr during transition is in a sense an act of resistance to the urge for connectivity. Tumblr enables separation from existing networks and rejection of traditional tiebased social connections.

In the early days of social network sites $\left(\mathrm{SNS}^{6}\right)$, Donath and boyd [28] discussed how SNSs, by making people's connections visible, removed some of the privacy barriers that people use in the physical world to separate incompatible social contexts. In the physical world, people separated social contexts with time and space; yet on SNSs, one's entire network was brought together into one digital space [28], causing context collapse [11].

Donath and boyd [28] suggested that people could segment their networks by using different SNSs as separate contexts, but that this would likely not be successful because one major benefit of SNSs is to have a set of diverse and heterogeneous connections and potential connections in one place. Instead, they proposed that a better solution would be to facet networks on one SNS by placing people into categories and posting content to selected groups of people [28]. Interestingly, in the years since, this strategy has been implemented by a variety of platforms, most notably on Facebook in the form of friend lists and groups. Yet, despite the ability to facet networks using friends lists on Facebook, most users do not use this feature because it is cumbersome and confusing and due to a lack of trust that the privacy settings will work as intended $[1,40,80]$.

Thus, instead of segmenting networks on one SNS, people often separate their networks by using different social media sites for different networks and purposes, as I have shown in this study, and as others have found [81,98]. Such separations are not necessary for every context; if a person wants to post different content for their gym friends vs. their co-workers, Facebook lists may work fine. However, during major life transitions and in stigmatized contexts, people often find it necessary to impose boundary regulation [63] by presenting different identities and sharing different content on two (or more) different social media sites.

The sites where people go to present stigmatized or transitional content and identities are often sites where connections are not traversable. In this paper's case study, people expressed transitional identities on Tumblr, a site that does not show network connections by default ${ }^{7}$. People also use sites like Reddit, on which users do not have any sort of friend or follower network, to discuss stigmatized identities and experiences [5]. Tumblr and Reddit, in addition to not showing visible connections, also enable people to create and use multiple accounts or profiles. In this way, Tumblr transition bloggers can have multiple blogs to segment their transition-related content and followers from, for example, their Pokémon obsession. Limiting networks can add value to those networks [28]. Part of the value of the Tumblr transition blog

\footnotetext{
${ }^{6}$ I make a distinction here between SNSs, on which people can "view and traverse their list of connections and those made by others within the system" [12], and social media sites more broadly, where people's networks are not necessarily visible to others (e.g., Tumblr).

${ }^{7}$ Tumblr users can display their followers to others by augmenting the code for their blog, but the default is for connections to be invisible.
} 
community, and other transitional communities, is that they provide a limited network that enables social transition machinery to work for a particular transitional context.

Given that the ability to present multiple identities on social media is a critical part of moving forward through life transitions, and that the ability to do so requires segmenting audiences, the idea of having one primary social media site on which to present oneself is antiquated, whether or not that site enables audience segmentation. Life transitions are not only a marginal state; every person experiences multiple life transitions throughout their lives, and sometimes several in tandem. Instead of a primary social media site that expects a static identity and a lifelong network, people require a series of separate pieces of social transition machinery that work together to facilitate life's transitions. Like cogs in a machine, these separate social media spaces work together but often do not intersect.

Technologists should resist uncritically espousing increased connection in online spaces and features that facilitate connection without accounting for context. While we certainly have the technological ability to link one's Facebook profile to one's Tumblr blog (e.g., through facial recognition or geographical or textual pattern matching), to do so would be both unethical and equivalent to throwing a wrench in the social transition machinery. Other attempts to connect people across sites, such as "people you may know" prompts, are useful in some contexts, such as if a person wishes to leverage personal connections to grow their professional network on LinkedIn. Yet an attempt to suggest connections to add to one's Tumblr network based on one's Facebook network would be deeply problematic, given the intentional separation that participants in this study described. Similarly, using Facebook or Google to log in to other sites across the Internet or mobile apps may inadvertently link sensitive or stigmatized identity facets to one's Facebook or Google profile and network. Connectivity can be powerful, and has been a revolutionary benefit of the Internet. Yet separation can also be powerful in enabling people to make life changes and discuss stigmatized identity facets. Social technology designers should consider the implications of connectivity, and the particular contexts in which technologies are used, when deciding whether and how sites and apps should connect with people's existing online social networks.

\section{CONCLUSION}

Existing on multiple social media sites simultaneously is central to many people's online social lives today [98]. However, presenting different versions of self to different networks is especially important for people during life transitions, as I have shown in this study. Transition work cannot be done all in one online place. My concept of social transition machinery describes the ways that different social media sites and networks remain separate, yet work together, to enable people to carry out different types of transition work and draw from different types of support networks. Using this concept, I contribute a new understanding of how people experience liminality on social media, and argue that reconstructing one's online identity on social media is a rite of passage. My description of liminality is a substantial departure from previous literature, which characterized transitional periods in people's lives as neutral or identity-less - which, in my view, further marginalizes transitional populations. Instead, I argue that existing multiply on different social media sites and remaining connected to several different networks is far from a neutral experience. To best facilitate online transition work, social media sites should be designed to foster social connectivity while acknowledging the importance of social media site separation. 


\section{ACKNOWLEDGMENTS}

Even though this is a single-authored paper, this work was done with help from many people. Thank you to the participants in the study, and to the bloggers whose data I analyzed in this research, for sharing their time and their data. I could not have done this work without them, and I acknowledge their generosity in allowing me, and the readers of this work, to learn from their experiences. Special thanks to research assistants Katrina Vergara for help with data collection and Gustavo Figueroa for help with interview transcription and analysis. Thanks to Gillian Hayes for support throughout the research process and valuable feedback on this work. I appreciated helpful feedback on various drafts of this work from Nazanin Andalibi, LouAnne Boyd, Phoebe Chua, Michael Dickard, Andrea Forte, Tim Gorichanaz, Gloria Mark, Kate Ringland, and Bonnie Ruberg. Finally, thank you to the anonymous reviewers and ACs. This work was funded in part by an NSF GRFP and a UCI James Harvey Scholar Award.

\section{REFERENCES}

[1] Alessandro Acquisti and Ralph Gross. 2006. Imagined Communities: Awareness, Information Sharing, and Privacy on the Facebook. In Privacy Enhancing Technologies, George Danezis and Philippe Golle (eds.). Springer Berlin Heidelberg, 36-58. http://link.springer.com/chapter/10.1007/11957454_3

[2] Alex A. Ahmed. 2018. Trans Competent Interaction Design: A Qualitative Study on Voice, Identity, and Technology. Interacting with Computers 30, 1: 53-71. https://doi.org/10.1093/iwc/iwx018

[3] Ban Al-Ani, Gloria Mark, and Bryan Semaan. 2010. Blogging in a region of conflict: supporting transition to recovery. In Proceedings of the SIGCHI Conference on Human Factors in Computing Systems, 1069-1078.

[4] Nazanin Andalibi and Andrea Forte. 2018. Announcing Pregnancy Loss on Facebook: A Decision-Making Framework for Stigmatized Disclosures on Identified Social Network Sites. In Proceedings of the 2018 CHI Conference on Human Factors in Computing Systems (CHI '18), 158:1-158:14. https://doi.org/10.1145/3173574.3173732

[5] Nazanin Andalibi, Oliver L. Haimson, Munmun De Choudhury, and Andrea Forte. 2016. Understanding Social Media Disclosures of Sexual Abuse Through the Lenses of Support Seeking and Anonymity. In Proceedings of the 2016 CHI Conference on Human Factors in Computing Systems (CHI '16), 3906-3918. https://doi.org/10.1145/2858036.2858096

[6] Greta R. Bauer, Jessica Braimoh, Ayden I. Scheim, and Christoffer Dharma. 2017. Transgender-inclusive measures of sex/gender for population surveys: Mixed-methods evaluation and recommendations. PLOS ONE 12, 5: e0178043. https://doi.org/10.1371/journal.pone.0178043

[7] Diana Beirl, Anya Zeitlin, Jerald Chan, Kai Ip Alvin Loh, and Xiaodi Zhong. 2017. GotYourBack: An Internet of Toilets for the Trans* Community. In Proceedings of the 2017 CHI Conference Extended Abstracts on Human Factors in Computing Systems, 39-45. https://doi.org/10.1145/3027063.3049272

[8] Rena Bivens. 2017. The gender binary will not be deprogrammed: Ten years of coding gender on Facebook. New Media \& Society 19, 6: 880-898. https://doi.org/10.1177/1461444815621527

[9] Rena Bivens and Oliver L. Haimson. 2016. Baking Gender Into Social Media Design: How Platforms Shape Categories for Users and Advertisers. Social Media + Society 2, 4: 2056305116672486. https://doi.org/10.1177/2056305116672486

[10] Anne Bolin. 1988. In Search of Eve: Transsexual Rites of Passage. Bergin \& Garvey.

[11] danah boyd. 2008. Taken Out of Context: American Teen Sociality in Networked Publics. University of California, Berkeley.

[12] danah m. boyd and Nicole B. Ellison. 2007. Social Network Sites: Definition, History, and Scholarship. Fournal of Computer-Mediated Communication 13, 1: 210-230. https://doi.org/10.1111/j.1083-6101.2007.00393.x

[13] Sarah Bratt, Bryan Semaan, Lauren Britton, Bryan Dosono, and Franco Zeno. 2017. Translation in Personal Crises: Opportunities for Wearables Design. 14.

[14] William Bridges. 2004. Transitions: Making Sense Of Life's Changes. Da Capo Press.

[15] Jed R. Brubaker, Gillian R. Hayes, and Paul Dourish. 2013. Beyond the Grave: Facebook as a Site for the Expansion of Death and Mourning. The Information Society 29, 3: 152-163. https://doi.org/10.1080/01972243.2013.777300

[16] Anne Bruce, Laurene Sheilds, Anita Molzahn, Rosanne Beuthin, Kara Schick-Makaroff, and Sheryl Shermak. 2014. Stories of Liminality: Living With Life-Threatening Illness. Fournal of Holistic Nursing 32, 1: 35-43. https://doi.org/10.1177/0898010113498823

[17] Amy S. Bruckman. 1993. Gender Swapping on the Internet. In Proceedings of INET'93.

[18] Moira Burke and Robert Kraut. 2013. Using Facebook After Losing a Job: Differential Benefits of Strong and Weak Ties. In Proceedings of the 2013 Conference on Computer Supported Cooperative Work (CSCW '13), 1419-1430. https://doi.org/10.1145/2441776.2441936 
[19] Peter J. Burke. 1991. Identity Processes and Social Stress. American Sociological Review 56, 6: 836-849. https://doi.org/10.2307/2096259

[20] Peter J. Burke. 2006. Identity Change. Social Psychology Quarterly 69, 1: 81-96.

[21] Judith Butler. 1999. Gender Trouble. Taylor \& Francis.

[22] Alexander Cho. 2015. Queer Reverb: Tumblr, Affect, Time. In Networked Affect. MIT Press.

[23] Avery Dame. 2013. "I'm your hero? Like me?": The role of 'expert' in the trans male vlog. Fournal of Language and Sexuality 2, 1: 40-69. https://doi.org/10.1075/jls.2.1.02dam

[24] Avery Dame. 2016. Making a name for yourself: tagging as transgender ontological practice on Tumblr. Critical Studies in Media Communication 33, 1: 23-37. https://doi.org/10.1080/15295036.2015.1130846

[25] Munmun De Choudhury and Michael Massimi. 2015. "She said yes!"-Liminality and Engagement Announcements on Twitter. Proceedings of iConference 2015.

[26] Michael A. DeVito, Jeremy Birnholtz, and Jeffery T. Hancock. 2017. Platforms, People, and Perception: Using Affordances to Understand Self-Presentation on Social Media. In Proceedings of the 2017 ACM Conference on Computer Supported Cooperative Work and Social Computing (CSCW '17), 740-754. https://doi.org/10.1145/2998181.2998192

[27] Judith Donath. 2014. The Social Machine: Designs for Living Online. The MIT Press.

[28] Judith Donath and danah boyd. 2004. Public displays of connection. BT Technology fournal 22, 4: 71-82.

[29] Bryan Dosono, Yasmeen Rashidi, Taslima Akter, Bryan Semaan, and Apu Kapadia. 2017. Challenges in Transitioning from Civil to Military Culture: Hyper-Selective Disclosure through ICTs. Proc. ACM Hum.-Comput. Interact 1, 2.

[30] Abigail C. Durrant, David S. Kirk, Diego Trujillo-Pisanty, and Sarah Martindale. 2018. Admixed Portrait: Design to Understand Facebook Portrayals in New Parenthood. In Proceedings of the 2018 CHI Conference on Human Factors in Computing Systems (CHI '18), 12:1-12:14. https://doi.org/10.1145/3173574.3173586

[31] Helen Rose Fuchs Ebaugh. 1988. Becoming an Ex: The Process of Role Exit. University of Chicago Press.

[32] Marty Fink and Quinn Miller. 2014. Trans Media Moments Tumblr, 2011-2013. Television \& New Media 15, 7: 611-626. https://doi.org/10.1177/1527476413505002

[33] Arnold van Gennep. 1909. The rites of passage. University of Chicago Press, Chicago.

[34] M. Glacken, G. Kernohan, and V. Coates. 2001. Diagnosed with hepatitis C: A descriptive exploratory study. International journal of nursing studies 38, 1: 107-116.

[35] Amy Gonzales and Nicole Fritz. 2017. Prioritizing Flexibility and Intangibles: Medical Crowdfunding for Stigmatized Individuals. In Proceedings of the 2017 CHI Conference on Human Factors in Computing Systems, 2371-2375. https://doi.org/10.1145/3025453.3025647

[36] Shannon Greenwood, Andrew Perrin, and Maeve Duggan. 2016. Social Media Update 2016. Pew Research Center: Internet, Science \& Tech. http://www.pewinternet.org/2016/11/11/social-media-update-2016/

[37] Oliver L. Haimson. 2018. The Social Complexities of Transgender Identity Disclosure on Social Media. University of California, Irvine.

[38] Oliver L. Haimson, Nazanin Andalibi, Munmun De Choudhury, and Gillian R. Hayes. 2017. Relationship breakup disclosures and media ideologies on Facebook. New Media \& Society. https://doi.org/10.1177/1461444817711402

[39] Oliver L. Haimson, Anne E. Bowser, Edward F. Melcer, and Elizabeth F. Churchill. 2015. Online Inspiration and Exploration for Identity Reinvention. In Proceedings of the 33rd Annual ACM Conference on Human Factors in Computing Systems (CHI '15), 3809-3818. https://doi.org/10.1145/2702123.2702270

[40] Oliver L. Haimson, Jed R. Brubaker, Lynn Dombrowski, and Gillian R. Hayes. 2015. Disclosure, Stress, and Support During Gender Transition on Facebook. In Proceedings of the 18th ACM Conference on Computer Supported Cooperative Work \& Social Computing (CSCW '15), 1176-1190. https://doi.org/10.1145/2675133.2675152

[41] Oliver L. Haimson, Jed R. Brubaker, Lynn Dombrowski, and Gillian R. Hayes. 2016. Digital Footprints and Changing Networks During Online Identity Transitions. In Proceedings of the 2016 CHI Conference on Human Factors in Computing Systems (CHI '16), 2895-2907. https://doi.org/10.1145/2858036.2858136

[42] Oliver L. Haimson and Anna Lauren Hoffmann. 2016. Constructing and enforcing "authentic" identity online: Facebook, real names, and non-normative identities. First Monday 21, 6. https://doi.org/10.5210/fm.v21i6.6791

[43] Foad Hamidi, Morgan Klaus Scheuerman, and Stacy M. Branham. 2018. Gender Recognition or Gender Reductionism?: The Social Implications of Embedded Gender Recognition Systems. In Proceedings of the 2018 CHI Conference on Human Factors in Computing Systems (CHI '18), 8:1-8:13. https://doi.org/10.1145/3173574.3173582

[44] Jack Harrison, Jaime Grant, and Jody L. Herman. 2012. A gender not listed here: Genderqueers, gender rebels, and otherwise in the National Transgender Discrimination Survey. LGBTQ Public Policy fournal at the Harvard Kennedy School 2, 1 .

[45] Blake Hawkins and Jack Giesking. 2017. Seeking ways to our transgender bodies, by ourselves: Rationalizing transgender-specific health information behaviors. Proceedings of the Association for Information Science and Technology 54, 1: 702-704. https://doi.org/10.1002/pra2.2017.14505401122

[46] Jana Herwig. 2009. Liminality and communitas in social media: The case of Twitter. In Internet: Critical. Internet Research, 7-10. 
[47] Laura Horak. 2014. Trans on YouTube Intimacy, Visibility, Temporality. TSQ: Transgender Studies Quarterly 1, 4: 572585. https://doi.org/10.1215/23289252-2815255

[48] Hsiu-Fang Hsieh and Sarah E. Shannon. 2005. Three Approaches to Qualitative Content Analysis. Qualitative Health Research 15, 9: 1277-1288. https://doi.org/10.1177/1049732305276687

[49] Sandy E. James, Jody L. Herman, Susan Rankin, Mara Keisling, Lisa Mottet, and Ma'ayan Anafi. 2016. The Report of the 2015 U.S. Transgender Survey. National Center for Transgender Equality, Washington, DC. http://www.transequality.org/sites/default/files/docs/USTS-Full-Report-FINAL.PDF

[50] Samantha Jaroszewski, Danielle Lottridge, Oliver L. Haimson, and Katie Quehl. 2018. "Genderfluid” or "Attack Helicopter": Responsible HCI Research Practice with Non-binary Gender Variation in Online Communities. In Proceedings of the 2018 CHI Conference on Human Factors in Computing Systems (CHI '18), 307:1-307:15. https://doi.org/10.1145/3173574.3173881

[51] Debbie Kralik. 2002. The quest for ordinariness: transition experienced by midlife women living with chronic illness. fournal of Advanced Nursing 39, 2: 146-154. https://doi.org/10.1046/j.1365-2648.2000.02254.x

[52] Debbie Kralik, Kate Visentin, and Antonia Van Loon. 2006. Transition: a literature review. fournal of Advanced Nursing 55, 3: 320-329. https://doi.org/10.1111/j.1365-2648.2006.03899.x

[53] Danielle Lottridge, Joy Kim, Joseph “Jofish” Kaye, and Nazanin Andalibi. Frontstage, Backstage, Midstage: Identity Performance on Social Media. under review.

[54] Haiwei Ma, C. Estelle Smith, Lu He, Saumik Narayanan, Robert A. Giaquinto, Roni Evans, Linda Hanson, and Svetlana Yarosh. 2017. Write for Life: Persisting in Online Health Communities Through Expressive Writing and Social Support. Proc. ACM Hum.-Comput. Interact. 1, CSCW: 73:1-73:24. https://doi.org/10.1145/3134708

[55] Clare Madge and Henrietta O’Connor. 2005. Mothers in the making? Exploring liminality in cyber/space. Transactions of the Institute of British Geographers 30, 1: 83-97. https://doi.org/10.1111/j.1475-5661.2005.00153.x

[56] Kristine Martin-McDonald and David Biernoff. 2002. Initiation into a dialysis dependent life: An examination of rites of passage. Nephrology Nursing fournal 29, 4: 347-353.

[57] Alice E. Marwick and danah boyd. 2010. I tweet honestly, I tweet passionately: Twitter users, context collapse, and the imagined audience. New Media \& Society 13, 1: 114-133. https://doi.org/10.1177/1461444810365313

[58] Carolyn R. Miller and Dawn Shepherd. 2004. Blogging as social action: A genre analysis of the weblog. Into the blogosphere: Rhetoric, community, and culture of weblogs 18, 1: 1-24.

[59] Wendy Moncur, Lorna Gibson, and Daniel Herron. 2016. The role of digital technologies during relationship breakdowns.

[60] Bonnie A. Nardi, Diane J. Schiano, and Michelle Gumbrecht. 2004. Blogging As Social Activity, or, Would You Let 900 Million People Read Your Diary? In Proceedings of the 2004 ACM Conference on Computer Supported Cooperative Work (CSCW '04), 222-231. https://doi.org/10.1145/1031607.1031643

[61] Abigail Oakley. 2016. Disturbing Hegemonic Discourse: Nonbinary Gender and Sexual Orientation Labeling on Tumblr. Social Media + Society 2, 3: 2056305116664217. https://doi.org/10.1177/2056305116664217

[62] Zizi Papacharissi. 2015. Toward New Journalism(s). fournalism Studies $16, \quad 1: \quad 27-40$. https://doi.org/10.1080/1461670X.2014.890328

[63] Sandra Petronio. 2002. Boundaries of Privacy: Dialectics of Disclosure. State University of New York Press, Albany.

[64] Rachel Premack. 2016. Tumblr's Depression Connection. The Ringer. https://theringer.com/tumblr-communitiesdepression-mental-illness-anxiety-c2ca927cd305\#.11etdnit6

[65] PyTumblr. PyTumblr. GitHub. https://github.com/tumblr/pytumblr

[66] Tobias Raun. 2010. Screen-Births: Exploring the Transformative Potential in Trans Video Blogs. Graduate fournal of Social Science 7, 2: 113-30.

[67] Tobias Raun. 2015. Video blogging as a vehicle of transformation: Exploring the intersection between trans identity and information technology. International fournal of Cultural Studies 18, 3: 365-378. https://doi.org/10.1177/1367877913513696

[68] Bryce J. Renninger. 2015. "Where I can be myself ... where I can speak my mind": Networked counterpublics in a polymedia environment. New Media \& Society 17, 9: 1513-1529. https://doi.org/10.1177/1461444814530095

[69] Kathryn E. Ringland. 2018. Exploring Virtual Worlds as a Support for Social Play in Children with Disabilities. University of California, Irvine.

[70] Brady Robards. 2012. Leaving MySpace, joining Facebook: 'Growing up' on social network sites. Continuum 26, 3: 385-398. https://doi.org/10.1080/10304312.2012.665836

[71] Corina Sas and Steve Whittaker. 2013. Design for forgetting: disposing of digital possessions after a breakup. In Proceedings of the 2013 ACM annual conference on Human factors in computing systems (CHI '13), 1823-1832. https://doi.org/10.1145/2466110.2466241

[72] 7Diane J. Schiano, Bonnie A. Nardi, Michelle Gumbrecht, and Luke Swartz. 2004. Blogging by the Rest of Us. In CHI '04 Extended Abstracts on Human Factors in Computing Systems (CHI EA '04), 1143-1146. https://doi.org/10.1145/985921.986009 
[73] Nancy K. Schlossberg. 1995. Counseling Adults in Transition: Linking Practice With Theory. Springer Publishing Company.

[74] Yukari Seko and Stephen P. Lewis. 2016. The self-harmed, visualized, and reblogged: Remaking of self-injury narratives on Tumblr. New Media \& Society: 1461444816660783. https://doi.org/10.1177/1461444816660783

[75] Bryan Semaan, Lauren M. Britton, and Bryan Dosono. 2017. Military Masculinity and the Travails of Transitioning: Disclosure in Social Media. In Proceedings of the 2017 ACM Conference on Computer Supported Cooperative Work and Social Computing (CSCW '17), 387-403. https://doi.org/10.1145/2998181.2998221

[76] Bryan C. Semaan, Lauren M. Britton, and Bryan Dosono. 2016. Transition Resilience with ICTs: "Identity Awareness" in Veteran Re-Integration. In Proceedings of the 2016 CHI Conference on Human Factors in Computing Systems (CHI '16), 2882-2894. https://doi.org/10.1145/2858036.2858109

[77] Bryan Semaan and Gloria Mark. 2011. Creating a Context of Trust with ICTs: Restoring a Sense of Normalcy in the Environment. In Proceedings of the ACM 2011 Conference on Computer Supported Cooperative Work (CSCW '11), 255264. https://doi.org/10.1145/1958824.1958863

[78] Bryan Semaan and Gloria Mark. 2012. "Facebooking” Towards Crisis Recovery and Beyond: Disruption As an Opportunity. In Proceedings of the ACM 2012 Conference on Computer Supported Cooperative Work (CSCW '12), 27-36. https://doi.org/10.1145/2145204.2145214

[79] Gail Sheehy. 2006. Passages: Predictable Crises of Adult Life. Ballantine Books.

[80] Manya Sleeper, Rebecca Balebako, Sauvik Das, Amber Lynn McConahy, Jason Wiese, and Lorrie Faith Cranor. 2013. The Post That Wasn't: Exploring Self-censorship on Facebook. In Proceedings of the 2013 Conference on Computer Supported Cooperative Work (CSCW '13), 793-802. https://doi.org/10.1145/2441776.2441865

[81] Manya Sleeper, William Melicher, Hana Habib, Lujo Bauer, Lorrie Faith Cranor, and Michelle L. Mazurek. 2016. Sharing Personal Content Online: Exploring Channel Choice and Multi-Channel Behaviors. In Proceedings of the 2016 CHI Conference on Human Factors in Computing Systems (CHI '16), 101-112. https://doi.org/10.1145/2858036.2858170

[82] Alix G. Sleight. 2016. Liminality and Ritual in Biographical Work: A Theoretical Framework for Cancer Survivorship. International Journal of Transpersonal Studies 35, 1: 52-61.

[83] Cooper Smith. 2013. Tumblr Offers Advertisers A Major Advantage: Young Users, Who Spend Tons Of Time On The Site. Business Insider. http://www.businessinsider.com/tumblr-and-social-media-demographics-2013-12

[84] Allucquère Rosanne Stone. 1995. The War of Desire and Technology at the Close of the Mechanical Age. MIT Press.

[85] Anselm Strauss and Juliet M. Corbin. 1998. Basics of Qualitative Research: Techniques and Procedures for Developing Grounded Theory. SAGE Publications.

[86] Susan Stryker. 2009. Transgender History. Seal Press.

[87] Bjørn Thomassen. 2009. The uses and meanings of liminality. International Political Anthropology 2, 1: 5-27.

[88] Tumblr. 2014. Application Developer and API License Agreement | Tumblr. https://www.tumblr.com/docs/en/api_agreement

[89] Tumblr. Tumblr API. https://www.tumblr.com/docs/en/api/v2

[90] Sherry Turkle. 1999. Cyberspace and Identity. Contemporary Sociology 28, 6: 643-648. https://doi.org/10.2307/2655534

[91] Victor Turner. 1969. The Ritual Process: Structure and Anti-Structure. Transaction Publishers.

[92] Victor Turner. 1987. Betwixt and Between: The Liminal Period in Rites of Passage. In Betwixt \& Between: Patterns of Masculine and Feminine Initiation. Open Court Publishing.

[93] Son Vivienne. 2017. "I will not hate myself because you cannot accept me": Problematizing empowerment and genderdiverse selfies. Popular Communication 15, 2: 126-140. https://doi.org/10.1080/15405702.2016.1269906

[94] Stephen Whittle. 1998. The Trans-Cyberian Mail Way. Social \& Legal Studies 7, 3: $389-408$. https://doi.org/10.1177/096466399800700304

[95] Mandy Wilson. 2002. 'I am the Prince of Pain, for I am a Princess in the Brain': Liminal Transgender Identities, Narratives and the Elimination of Ambiguities. Sexualities 5, 4: 425-448. https://doi.org/10.1177/1363460702005004003

[96] Jiejun Xu, Ryan Compton, Tsai-Ching Lu, and David Allen. 2014. Rolling through tumblr: characterizing behavioral patterns of the microblogging platform. In WebSci, 13-22. https://doi.org/10.1145/2615569.2615694

[97] Shanyang Zhao, Sherri Grasmuck, and Jason Martin. 2008. Identity construction on Facebook: Digital empowerment in anchored relationships. Computers in Human Behavior 24, 5: 1816-1836. https://doi.org/10.1016/j.chb.2008.02.012

[98] Xuan Zhao, Cliff Lampe, and Nicole B. Ellison. 2016. The Social Media Ecology: User Perceptions, Strategies and Challenges. In Proceedings of the 2016 CHI Conference on Human Factors in Computing Systems (CHI '16), 89-100. https://doi.org/10.1145/2858036.2858333

Received April 2018; revised July 2018; accepted November 2018

Proceedings of the ACM on Human-Computer Interaction, Vol. 2, No. CSCW, Article 63, Publication date: November 2018. 